\title{
Cutaneous hypersensitivity reactions due to thiacetazone in the treatment of tuberculosis in Zambian children infected with HIV-I
}

\author{
Chifumbe Chintu, Chewe Luo, Ganapati Bhat, Mario Raviglione, Herbert DuPont, \\ Alimuddin Zumla
}

\begin{abstract}
Tuberculosis is one of the most common infections in Zambian adults and children infected with HIV. In Africa, cutaneous hypersensitivity reactions attributed to thiacetazone during treatment of tuberculosis in adults infected with HIV-I have been well documented. This study monitored adverse drug reactions during treatment for tuberculosis over an 18 month period (1 April 1990 to 31 October 1991) in 237 children with a clinical diagnosis of tuberculosis (125 boys and 112 girls; 88/237 (37\%) infected with HIV-I) and 242 control children (149 boys and 93 girls; $26 / 242(11 \%)$ infected with HIV-I). Twenty two (9\%) of the 237 children with tuberculosis developed hypersensitivity skin reactions during the course of treatment. Adverse skin reactions were seen more often in children infected with HIV than in those who were not (odds ratio 11.65, 95\% confidence interval 3.07 to 34.88$)$. These represented $19(21 \%)$ of 88 children infected with HIV and three (2\%) of 149 children not infected with HIV. These skin reactions occurred after a period of treatment ranging between two and four weeks among 14 children receiving the HST (isoniazid, streptomycin, thiacetazone) regimen and eight children receiving the HSTR (isoniazid, streptomycin, thiacetazone, rifampicin) regimen. Twelve (55\%) of the 22 children who reacted adversely to treatment developed the Stevens-Johnson syndrome. All 12 of these children with the Stevens-Johnson syndrome were infected with HIV. The mortality among these children who developed the StevensJohnson syndrome was $91 \%$ (11 of 12 died within three days of the onset of the reaction). No further reactions were observed in the $\mathbf{1 1}$ children who recovered from the cutaneous hypersensitivity reactions after thiacetazone was discontinued over a period of six months of further treatment for tuberculosis. The results of this study were in part responsible for the recommendations put forward by the World Health Organisation to avoid the use of thiacetazone in the treatment of tuberculosis in children infected with HIV.

(Arch Dis Child 1993; 68: 665-668)
\end{abstract}

The relation between the HIV infection and tuberculosis in Africa has been well documented. ${ }^{1-5}$ In Zambia an exponential increase in the number of reported cases of tuberculosis has occurred over the past seven years ${ }^{6}$ and epidemiological information from studies in adults indicates that this is most probably linked to the HIV-I epidemic. ${ }^{7}$ Owing to its low cost and ease of administration, thiacetazone has been one of the cheapest and most commonly used drugs in the treatment of tuberculosis in Africa, although it is not without side effects. Well before the AIDS epidemic, regimens containing streptomycin, thiacetazone, and isoniazid were reported to be associated with cutaneous hypersensitivity reactions, while hypersensitivity to thiacetazone was thought to be responsible for $40 \%$ of skin rashes in patients with tuberculosis. ${ }^{89}$ From studies of adult patients with tuberculosis in Africa, serious and occasionally fatal drug reactions in patients infected with HIV have been reported by several workers. ${ }^{6710-12}$ Information on the incidence of adverse reactions to treatment for tuberculosis in children infected with HIV is lacking, however. We prospectively evaluated the incidence of cutaneous hypersensitivity reactions as part of an ongoing cohort study of children with tuberculosis who were positive and negative for antibody to HIV.

\section{Subjects and methods}

SUBJECTS

All consecutive newly diagnosed cases of tuberculosis in children from 1 month to 15 years of age seen at the paediatric department of the University Teaching Hospital in Lusaka, Zambia, between 1 April 1990 and 31 October 1991 were asked to enrol in the study. Informed consent was obtained and pretest counselling for HIV offered.

DEFINITION OF TUBERCULOSIS

The diagnosis of tuberculosis was defined as the presence of three of the following: (a) symptoms and signs suggestive of tuberculosis; (b) sputum smear or gastric washings positive for acid fast bacilli; (c) lymph node or other tissue biopsy indicative of tuberculosis; (d) radiological features suggestive of tuberculosis; (e) response to treatment for tuberculosis; (f) history of close contact with a patient with tuberculosis; and (g) positive skin reaction to testing with purified protein derivative.

TREATMENT REGIMEN

In accordance with national guidelines at the time, treatment of localised tuberculosis was initiated with the HST regimen (isoniazid $10-15 \mathrm{mg} / \mathrm{kg} /$ day by mouth, streptomycin $20-40$
University Teaching $H$

Zambia.

Accepted 16 December 1992 
$\mathrm{mg} / \mathrm{kg} /$ day intramuscularly for two months, thiacetazone $5-8 \mathrm{mg} / \mathrm{kg} /$ day by mouth) for a minimum period of 12 months. In disseminated forms of the disease (tuberculous meningitis, miliary tuberculosis at initiation, and in patients with localised disease where no response to treatment was seen after one month of HST, the HSTR regimen (HST plus rifampicin $15-20 \mathrm{mg}$ / $\mathrm{kg}$ /day) was used for a minimum period of six months.

\section{HIV-I SEROLOGY}

HIV-I serology was determined using two enzyme linked immunosorbant assays (ELISAs), Welcozyme (Wellcome Diagnostics, Dartford, Oxford) and the antiglobulin recombinant ELISA (DuPont de Nemours, Wilmington, Delaware, USA). Those samples postive by the two ELISAs were subjected to western blot analysis. HIV-II has not been reported to be important in Zambia. In children less than 18 months of age the HIV serology was repeated when the child was 18 months old.

\section{DEFINITION OF HYPERSENITIVITY}

Cutaneous hypersensitivity reactions to treatment for tuberculosis were defined as a skin rash appearing after the start of treatment with or without mucosal involvement or the presence of itching, and for those who survived, the disappearance of the rash on withdrawal of thiacetazone. Lesions were classified as maculopapular, target, or mucosal, and the presence of desquamation or bullae was noted. The clinical disorder was classified as Stevens-Johnson syndrome when the patient was systemically ill with the mucosal surfaces affected (mouth, eye, urinary tract, and vagina) with extensive skin disease and the presence of bullae or target lesions.

\section{MANAGEMENT OF SIDE EFFECTS}

When side effects were noted all drugs were stopped and prednisolone and chlorpheniramine were prescribed at the discretion of the doctor in charge. In those patients who recovered, thiacetazone was discontinued on the recommencement of treatment for tuberculosis, and replaced by rifampcin in those receiving the HST regimen and by pyrizinamide in those

Table 1 Age, sex distribution, and HIV status of children with Stevens-fohnson syndrome

\begin{tabular}{ccllll}
\hline $\begin{array}{l}\text { Patient } \\
\text { No }\end{array}$ & Age & Sex & $\begin{array}{l}\text { HIV } \\
\text { status }\end{array}$ & Treatment & Outcome \\
\hline 1 & 5 months & M & + & HST & Died \\
2 & 8 months & M & + & HST & Died \\
3 & 11 months & M & + & HST & Died \\
4 & 12 months & M & + & HST & Died \\
5 & 13 months & F & + & HSTR & Died \\
6 & 14 months & M & + & HSTR & Died \\
7 & 20 months & F & + & HST & Died \\
8 & 20 months & M & + & HST & Died \\
9 & 2 years & F & + & HSTR & Died \\
10 & 5 years & F & + & HSTR & Full recovery \\
11 & 5 years & F & + & HSTR & Died \\
12 & 6 years & M & + & HSTR & Died \\
\hline
\end{tabular}

*HST: $H=$ isoniazid, $S=$ streptomycin, and $T=$ thiacetazone. HSTR: $\mathrm{H}=$ isoniazid, $\mathrm{S}=$ streptomycin, $\mathrm{T}=$ thiacetazone, and $\mathbf{R}=$ rifampicin. receiving the HSTR regimen. Owing to the serious nature of the side effects encountered, no challenge test with individual drugs was undertaken.

\section{DATA RECORDING}

Clinical data were recorded on specially designed study forms. Children with tuberculosis positive and negative for antibodies to HIV were discharged after a clinical response to treatment was noted (clinical and radiological improvement, regression of lymph node size, and general well being) and then followed up at four weekly intervals at the outpatient clinics.

\section{DATA ANALYSIS}

The data obtained were analysed using the EPIINFO and EGRET software programs. ${ }^{1314}$

\section{Results}

\section{INCIDENCE OF CUTANEOUS HYPERSENSITIVITY} REACTIONS

This study monitored cutaneous hypersensitivity drug reactions during treatment for tuberculosis over an 18 month period in 237 children $(88 / 237$ (37\%) infected with HIV-I) with a clinical diagnosis of tuberculosis (125 boys and 112 girls). Twenty two (9\%) of 237 children developed adverse skin reactions to treatment for tuberculosis. These reactions were seen more often among children positive for antibody to HIV than in children negative for the antibody (odds ratio $11.65,95 \%$ confidence interval 3.07 to $34 \cdot 88$ ) affecting $19(21 \%)$ of 88 children infected with HIV and only three (2\%) of 149 children not infected with HIV.

\section{TIME OF ONSET}

These skin reactions occurred after a period of treatment ranging between two and four weeks of the onset of treatment, occurring in 14 children receiving the HST regimen (isoniazid, streptomycin, thiacetazone) and in eight children receiving the HSTR regimen (isoniazid, streptomycin, thiacetazone, and rifampicin).

\section{CLINICAL FEATURES OF REACTIONS}

In all children itching preceded the rash. Cutaneous reactions were extensive, bilateral, and maculopapular in nature. The more serious lesions were found in systemically ill children with mucocutaneous lesions, bullae and target lesions, and exfoliation of the skin (table 1). Of the 22 children who developed cutaneous reactions, $12(55 \%)$ had the Stevens-Johnson syndrome. All 12 children with the StevensJohnson syndrome were positive for antibody to HIV-I. The mortality among children who developed the Stevens-Johnson syndrome was $92 \%$ ( 11 of 12 died within three days of the reaction). Seven of the 10 children who developed only maculopapular rashes were infected with HIV (table 2). All 10 children with skin rashes recovered fully. After thiacetazone was discontinued from the treatment regimens, 
Table 2 Age, sex distribution, and HIV status of children with a predominantly maculopapular skin rash

\begin{tabular}{llllll}
\hline $\begin{array}{l}\text { Patient } \\
\text { No }\end{array}$ & Age & Sex & $\begin{array}{l}\text { HIV } \\
\text { status }\end{array}$ & Treatment & Outcome \\
\hline 13 & 10 months & M & + & HST & Full recovery \\
14 & 15 month & M & + & HST & Full recovery \\
15 & 13 months & M & - & HST & Full recovery \\
16 & 3 years & F & + & HSTR & Full recovery \\
17 & 6 years & M & + & HST & Full recovery \\
18 & 7 years & F & + & HST & Full recovery \\
19 & 9 years & M & - & HST & Full recovery \\
20 & 9 years & M & + & HST & Full recovery \\
21 & 9 years & F & + & HST & Full recovery \\
22 & 13 years & F & - & HST & Full recovery \\
\hline
\end{tabular}

${ }^{\star} \mathrm{HST}$ : $\mathrm{H}=$ isoniazid, $\mathrm{S}=$ streptomycin, and $\mathrm{T}=$ thiacetazone. HSTR: $H=$ isoniazid, $S=$ streptomycin, $T=$ thiacetazone, and $\mathrm{R}=$ rifampicin.

no further reactions were observed over a period of six months in those 11 children who recovered from the hypersensitivity reactions. Owing to the serious nature of the hypersensitivity reactions a challenge test with individual drugs was not undertaken.

\section{RISK FACTOR ANALYSIS}

The following clinical features at enrollment were not related to the subsequent development of hypersensitivity reactions: age, sex, weight, lymph node enlargement, splenomegaly, extent of tuberculosis, type of tuberculosis, and absolute lymphocyte counts.

\section{Discussion}

It is now well documented that in adult patients infected with HIV and tuberculosis there is a high mortality rate and that there is a disturbingly high frequency of toxic reactions attributed to thiacetazone..$^{1-7} 1011$ The most important are serious skin reactions, for which thiacetazone has often been implicated as the causative agent. Earlier work with thiacetazone suggested that its side effects were dose related. ${ }^{15}$ Evidence that the use of thiacetazone is associated with an increased morbidity and mortality among patients infected with HIV is mounting. ${ }^{70-12} \mathrm{~A}$ study of adult patients in Kenya showed that 22 (20\%) of 111 adult patients positive for antibody to HIV had cutaneous hypersensitivity reactions while receiving treatment for tuberculosis compared with $2(1 \%)$ of 176 patients negative for antibody to HIV. ${ }^{10}$ That study reported three deaths from toxic epidermal necrolysis, all occurring among the patients positive for antibody to HIV. On challenge no reactions were observed after isoniazid and streptomycin but six of seven patients reacted with thiacetazone. A study of adult patients with tuberculosis in Zambia reported Stevens-Johnson syndrome occurring in eight (2\%) of $346^{\circ}$ patients, all of whom were positive for antibody to HIV.?

This study has shown that children infected with HIV have an increased risk for the development of cutaneous hypersensitivity reactions to treatment for tuberculosis. The striking feature in our study was the frequency and severity of adverse reactions in children positive for antibody to HIV. We detected a high occurrence of Stevens-Johnson syndrome (12 (55\%) of 22 patients who developed hypersensitivity reactions) and a high mortality from this side effect $(92 \%)$ occurred. Although thiacetazone is a well recognised cause of adverse cutaneous reactions, ${ }^{16}$ with the use of multidrug treatment it is always difficult to incriminate with confidence any particular agent when side effects are noted. Owing to the high mortality associated with the side effects seen, we did not perform a test challenge to establish the offending drug. We assumed that thiacetazone was the offending drug as no further reactions were seen in patients who survived the hypersensitivity reactions and then received a regimen free from thiacetazone. The reactions described in this study are those we were able to detect in hospital or at the outpatient clinic. Owing to the poor follow up attendance of these patients (only $50 \%$ attended the third follow up at three months; Luo et al, unpublished data), it is likely that a good number who might have had reactions at home were not brought to our attention and thus could not be included in the analysis.

The underlying pathophysiological mechanisms responsible for the development of the Stevens-Johnson syndrome are not clear. Importantly, there appears no adequate explanation as to why only certain subjects positive for antibody to HIV should react so adversely to thiacetazone. The identification of clinical or immunological characteristics of patients who have experienced adverse reactions to thiacetazone that differentiate them from other patients infected with HIV may be of paramount importance. This analysis could help resource poor countries to identify groups at risk and tailor treatment regimens which contain thiacetazone for their patients infected with HIV.

Our observations in the treatment of tuberculosis in children infected with HIV and those of other studies in adults ${ }^{70-12}$ have important implications for tuberculosis treatment policies in areas where HIV is increasing in prevalence. The tuberculosis unit of the World Health Organisation (WHO) has issued guidelines for the treatment of tuberculosis in adults and children in national tuberculosis programmes. The data obtained in our study were in part responsible for the WHO's strong warning against the use of thiacetazone in patients at increased risk of HIV infection. ${ }^{6}$ These guidelines state that when possible, for those patients with newly diagnosed tuberculosis, the optimum six month regimen should be HRZE (isoniazid, rifampicin, pyrizinamide, ethambutol) for two months followed by HR for four months; or alternatively the eight month regimen (HRZE for two months followed by HE for six months, where thiacetazone is replaced by ethambutol in the continuation phase should be adopted. ${ }^{12}$ These guidelines further state that where the 12 month regimen used by us in this study is still in use (two months of HST followed by 10 months of HT), thiacetazone should be replaced by ethambutol. It is important to realise, however, that thiacetazone is the cheapest accompaniment to isoniazid in resource poor countries. Many countries with a high prevalence of HIV that currently use thiacetazone in regimens for the treatment of tuberculosis lack the funds to 
replace this drug. In these countries the following warning issued in the WHO guidelines must be heeded by all health care providers. If thiacetazone must be used all patients must be warned about the possibility of severe skin reactions and they must be advised to discontinue thiacetazone if these occur and to report immediately to their health care providers. Desensitisation for patients developing hypersensitivity reactions to thiacetazone should not be attempted. At the University Teaching Hospital in Lusaka, due to the high incidence of serious cutaneous hypersensitivity found in our study, thiacetazone has been omitted from treatment regimens since 1991 and patients with tuberculosis are now being treated with HSRZ (isoniazid, streptomycin, rifampicin, pyrazinamide). The efficacy of this regimen and its side effects are currently under evaluation.

This study was supported by the World Health Organisation grant A20-181-226 and a US National Institutes of Health, NIAID grant RO1 Al 31356-01A2.

1 Gilks CF, Brindle RJ, Otieno LS, et al. Extrapulmonary and disseminated tuberculosis in HIV-1 seropositive patients presenting to the acute medical services in Nairobi. AIDS $1990 ; 4: 981-5$.

2 Harries AD. Tuberculosis and HIV infection in developing countries. Lancet 1990; 335: 387-90.

3 DeCock KM, Gnaore E, Braun M, et al. Risk of tuberculosis in patients with HIV-I and HIV-II infections in Abidjan, Ivory Coast. BMF 1991; 302: 496-9.

4 Coleblunders RL, Ryder RW, Nzilambi N, et al. HIV infection in patients with tuberculosis in Kinshasha, Zaire. Am Rev Respir Dis 1989; 139: 1082-5.

5 Raviglione MC, Narain JP, Kochi A. HIV-associated tuberculosis in developing countries: clinical features, diagnosis and treatment. Bull World Health Organ 1992; 70. 515-26.

6 Tuberculosis/HIV research. Report of a WHO review and lanning meeting Geneva, 24-26 February 1992. Geneva:

7 Elliott AM, Luo NP, Tembo G, et al. Impact of HIV on tuberculosis in Zambia: a cross sectional study. BMf 1990 301: 412-5.

8 Ferguson C, Nunn A, Fox W, Miller A, Robinson D, Tall R $A$ second international co-operative investigation into thiacetazone side effects. Tubercle 1971; 52: 160-81.

9 Miller A, Fox W, Tall R. An international cooperative investigation into thiacetazone side effects. Tubercle 1966; 47: 33-74.

10 Nunn $P$, Kibuga D, Gathua $S$, et al. Cutaneous hypersensitivity reactions due to thiacetazone in HIV-1 serosensitivity reactions due to thiacetazone in HIV-1 sero-
positive patients treated for tuberculosis. Lancet 1992; 339:

11 Wirima JJ, Harries AD. Stevens-Johnson syndrome during anti-tuberculosis chemotherapy in HIV-seropositive patients: report on six cases. East Afr Med $\mathcal{F} 1991 ; 68: 64-6$.

12 Report 1992: severe hypersensitivity reactions among HIV eropositive patients with tuberculosis treated with thiceta zone. Whly Epidemiol Rec 1992; 67: 1-3

13 Dean AG, Dean JA, Burton AH, Dicker RC. Epi Info version 5; a word processing database and statistics program for epidemiology on microcomputers. Atlanta: Centers for Disease Control, 1980 .

14 EGRET: epidemiological, graphics, estimation and testing package. Seattle: Statistics and Epidemiology Research

15 Pearson CA. Thiacetazone toxicity in the treatment of tuberculosis patients in Nigeria. F Trop Med Hyg 1978; 8: 238-42.

16 Girling DJ. Adverse effects of antituberculosis drugs. Drugs 